\title{
HIF2 $\alpha$ in the uterine stroma permits embryo invasion and luminal epithelium detachment
}

\author{
Leona Matsumoto, ${ }^{1}$ Yasushi Hirota, ${ }^{1,2}$ Tomoko Saito-Fujita, ${ }^{1}$ Norihiko Takeda, ${ }^{3}$ Tomoki Tanaka,, ${ }^{1}$ Takehiro Hiraoka,, ${ }^{1}$ Shun Akaeda, ${ }^{1}$ \\ Hidetoshi Fujita, ${ }^{4}$ Ryoko Shimizu-Hirota, ${ }^{5}$ Shota Igaue, ${ }^{1}$ Mitsunori Matsuo, ${ }^{1}$ Hirofumi Haraguchi, ${ }^{1}$ Mayuko Saito-Kanatani, \\ Tomoyuki Fujii, ${ }^{\text {and Yutaka Osuga' }}$
}

'Department of Obstetrics and Gynecology, Graduate School of Medicine, The University of Tokyo, Bunkyo-ku, Tokyo, Japan. ${ }^{2}$ Precursory Research for Innovative Medical Care (PRIME), Japan Agency for Medical Research and Development (AMED), Bunkyo-ku, Tokyo, Japan. ${ }^{3}$ Department of Cardiovascular Medicine, Graduate School of Medicine, The University of Tokyo, Bunkyo-ku, Tokyo, Japan. ${ }^{4}$ Department of Future Medical Science, Institute of Medical Science, Tokyo Medical University, Shinjuku-ku, Tokyo, Japan. 드epartment of Internal Medicine, Center of Preventive Medicine, School of Medicine, Keio University, Shinjuku-ku, Tokyo, Japan.

\begin{abstract}
Although it has been reported that hypoxia inducible factor $2 \alpha$ (Hif2a), a major transcriptional factor inducible by low oxygen tension, is expressed in the mouse uterus during embryo implantation, its role in pregnancy outcomes remains unclear. This study aimed to clarify functions of uterine HIF using transgenic mouse models. Mice with deletion of Hif2a in the whole uterus (Hif2a-uKO mice) showed infertility due to implantation failure. Supplementation with progesterone $\left(\mathrm{P}_{4}\right)$ and leukemia inhibitory factor (LIF) restored decidual growth arrest and aberrant position of implantation sites in Hif2a-uKO mice, respectively, but did not rescue pregnancy failure. Histological analyses in Hif2a-uKO mice revealed persistence of the intact luminal epithelium, which blocked direct contact between stroma and embryo, inactivation of PIЗK-AKT pathway (embryonic survival signal), and failed embryo invasion. Mice with stromal deletion of Hif2a (Hif2a-sKO mice) showed infertility with impaired embryo invasion and those with epithelial deletion of Hif2a (Hif2a-eKO mice) showed normal fertility, suggesting the importance of stromal HIF2a in embryo invasion. This was reflected in reduced expression of membrane type 2 metalloproteinase (MT2-MMP), lysyl oxidase (LOX), VEGF, and adrenomedullin (ADM) in Hif2a-uKO stroma at the attachment site, suggesting that stromal HIF2 $\alpha$ regulates these mediators to support blastocyst invasion. These findings provide new insight that stromal HIF2 $\alpha$ allows trophoblast invasion through detachment of the luminal epithelium and activation of an embryonic survival signal.
\end{abstract}

\section{Introduction}

Infertility is a worldwide issue estimated to affect up to $30 \%$ of couples in both developed and developing countries (1). Infertile patients undergoing in vitro fertilization and embryo transfer (IVFET) are on the rise, and many of them experience recurrent implantation failure, which is a major problem in IVF-ET programs. The process of embryo implantation spanning blastocyst apposition and attachment with the uterine epithelium, along with invasion of blastocyst trophectoderm into the endometrium, is executed by molecular interactions between the embryo and uterus (2-5). Among others, progesterone $\left(\mathrm{P}_{4}\right)$ signaling, heparin-binding EGFlike growth factor (HB-EGF), and leukemia inhibitory factor-STAT3 (LIF-STAT3) signaling were reported as key molecular mediators of embryo apposition and attachment (6-11). However, other key molecules and pathways in these processes remain elusive.

Hypoxia inducible factor (HIF) is a major transcription factor that responds to low oxygen tension and induces the expression of hypoxia-related genes such as VEGF and erythropoietin (12). Through the induction of these genes, HIF is involved in vari-

Authorship note: LM, YH, and TSF contributed equally to this work. Conflict of interest: The authors have declared that no conflict of interest exists. Submitted: November 29, 2017; Accepted: May 1, 2018

Reference information: / Clin Invest. 2018;128(7):3186-3197.

https://doi.org/10.1172/JCI98931. ous physiological responses such as metabolism, cell death, and angiogenesis (13). HIF is composed of 2 subunits, $\alpha$ and $\beta$ (14). The HIF $\alpha$ subunit has 3 isotypes, HIF $1 \alpha$, HIF $2 \alpha$, and HIF $3 \alpha$, whereas the HIF $\beta$ subunit has 2 types, ARNT1 and ARNT2 (14). A previous study showed that the expression of Hifla is primarily in the uterine luminal epithelium during the peri-implantation period, and the expression of Hif $2 a$ is mainly in the stroma at the same time, whereas Hif $3 a$ is undetectable in the uterus (15). Notably, Hif $2 a$ is strongly expressed in the uterine stroma after embryo attachment (15). Based on expression patterns, we speculated that HIF $\alpha$ has functional roles in embryo implantation. Hifla and Hif $2 a$ homozygous null embryos die during gestation $(16,17)$, impeding functional analysis of HIF in the reproductive system. To overcome this difficulty, we generated mice with uterine tissue-specific deletion of Hifla and Hif2a using Pgr-Cre and Ltf-Cre drivers as previously described (18-21), and examined critical pregnancy events. Mice with Hif $2 a$ deletion in the entire uterus (Hif $2 a$-uKO mice) were infertile, whereas mice with uterine deletion of Hifla (Hifla-uKO mice) showed subfertility, indicating the importance of uterine $\mathrm{HIF} \alpha$ in fertility. The luminal epithelial barrier surrounding the embryo disappears just before embryo invasion in the littermate controls, whereas it persists and prevents embryo invasion in $H$ if $2 a$-uKO mice, suggesting impairment of this invasion process as a cause of infertility in Hif $2 a$-uKO mice. Importantly, detachment of luminal epithelium from stroma, which permits direct contact 
A

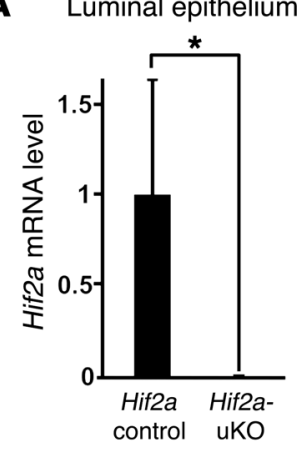

C

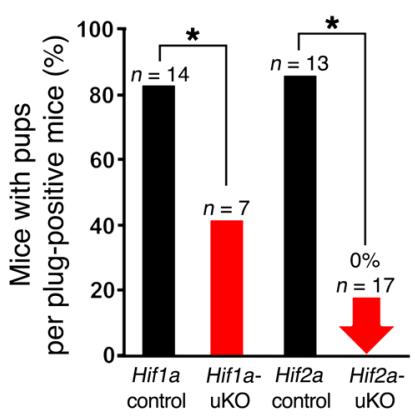

B

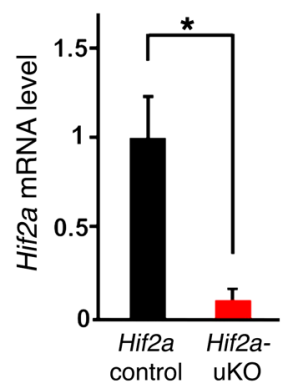

D

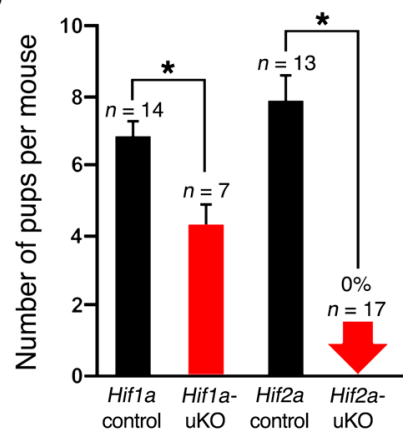

between stroma and embryo, and activation of PI3K-AKT pathway as an embryonic survival signal are not observed in Hif2a-uKO mice (unlike the littermate controls), providing evidence for a role of HIF2 $\alpha$ in embryo invasion. Our findings offer insights that uterine HIF2 $\alpha$ is critical for embryo invasion through activation of an embryo survival signal and detachment of the luminal epithelium.

\section{Results}

Mice with uterine deletion of Hif2a show infertility and those with uterine deletion of Hifla show subfertility. To explore the role of uterine HIF in pregnancy, we generated females with deletion of Hifla and Hif $2 a$ in the entire uterus (Hifla-uKO and Hif $2 a$-uKO, respectively) by crossing Hifla- or Hif $2 a$-loxP mice with Pgr-Cre mice. We first confirmed that HIF1 $\alpha$ and HIF $2 \alpha$ mRNA levels were significantly reduced in the uterus of Hifla-uKO and Hif2a-uKO mice, respectively (Figure 1, A and B and Supplemental Figure 1; supplemental material available online with this article; https://oi.org/10.1172/ JCI98931DS1). Hifla-uKO mice, Hif2a-uKO mice, and their littermate controls (Hifla control, Hif2a control) were mated with WT fertile male mice. We found that approximately $50 \%$ of the plugpositive Hifla-uKO mice did not deliver any pups and the number of pups with Hifla-uKO dams was significantly reduced (Figure 1, $\mathrm{C}$ and D). Surprisingly, we found that Hif2a-uKO mice were infertile (Figure 1, C and D), suggesting that uterine Hifa, especially Hif $2 a$, is critical for successful pregnancy. Based on these results, we focused on the role of Hif2a in the uterus in the subsequent analyses by using $H i f 2 a$-uKO mice.

Hif2a-uKO mice showing normal ovulation and preimplantation embryo development undergo impaired embryo implantation. We examined ovulation and fertilization in Hif2a-uKO mice by flushing eggs and/or embryos on day 2 of pregnancy (day $1=$ vaginal plug), and found both processes to be normal (Figure 2, A-C). We

Figure 1. Hif2a-uKO mice show infertility, and Hif1a-uKO mice show subfertility. (A and B) Hif2a mRNA levels were reduced in the uterine luminal epithelium and stroma of Hif2a-uKO mice, respectively $(n=4)$. (C and D) Hif1a-uKO mice were subfertile, and Hif2a-uKO mice were infertile. Hif1auKO mice, Hif2a-uKO mice, and their littermate controls (Hif1a control, Hif2a control) were mated with WT fertile male mice. (A, B, D) Mean \pm SEM, Student's $t$ test. (C) Fisher's exact probability test. In all panels, ${ }^{*} P<0.05$.

also confirmed normal development of preimplantation embryos and their timely transport from the oviduct into uterine lumens of Hif $2 a$-uKO mice by recording the number of blastocysts retrieved through flushing the uteri on day 4 of pregnancy (Figure 2, D and E). Successful implantation is the result of reciprocal interactions between the blastocyst and the receptive uterus. Stromal edema on day 4 morning leads to uterine luminal closure, placing the blastocyst in close apposition with the luminal epithelium. We previously reported that proliferation-to-differentiation switching, cessation of epithelial proliferation, and acceleration of stromal proliferation in the uterus on day 4 morning are hallmarks of uterine receptivity in embryo implantation (7). Immunostaining of Ki67, a cell proliferation marker, revealed that uteri of Hif2a-uKO mice had normal proliferation-to-differentiation switching, comparable to the controls (Supplemental Figure 2, A-C). Embryo apposition is followed by intimate adherence of the blastocyst trophectoderm to the luminal epithelium (attachment reaction), marking the first discernible sign of implantation from midnight of day 4 to day 5 morning. The attachment reaction coincides with increased stromal vascular permeability at the site of the blastocyst and can be demarcated by distinct blue bands along the uterus after intravenous injection of Chicago blue dye solution (8). We found that the number of distinct implantation sites in Hif2a-uKO mice was comparable to Hif2a controls on day 5 morning (Figure 2, F and G). With the implantation process in progress, stromal cells surrounding the implanting embryo undergo decidualization. The luminal epithelium surrounding the implantation sites disappears on day 5 evening, and embryo invasion into the stroma starts from day 5 night to day 6 morning. Decidual growth peaks on day 8 of pregnancy. To determine whether this process is normal in Hif $2 a$-uKO mice, we counted the number of implantation sites in Hif2a-uKO mice and the controls on days 6 and 8 of pregnancy. The number of implantation sites remained comparable between the 2 groups on day 6 (Figure 2, $\mathrm{H}$ and I), but normal implantation sites with decidualization completely disappeared in Hif $2 a$-uKO mice on day 8 (Figure 2, J and K). By H\&E staining, we confirmed that embryos were completely degraded and decidualization was impaired in Hif2auKO mice on day 8 of pregnancy (Figure 2L), suggesting decidual growth arrest and implantation failure in Hif $2 a-u K O$ mice.

$\mathrm{P}_{4}$ administration rescues decidualization in Hif $2 a-u K O$ mice but does not rescue pregnancy failure. It has been reported that murine decidua-derived factors serve as luteotrophins to prolong the life span of the corpus luteum and maintain luteal $\mathrm{P}_{4}$ production (22-24). Prolactin-related factors Prl3c1 and Prlr are hallmarks of decidual health and contribute to activation of the corpus luteum and ovarian secretion of $\mathrm{P}_{4}$ in rodents (25-31). In Hif $2 a$-uKO mice, the expression of Prl3c1 and Prlr was significantly reduced (Figure 3, A-C), suggesting that unhealthy decidual condition compromises luteotrophy in Hif2a-uKO mice. As 
A

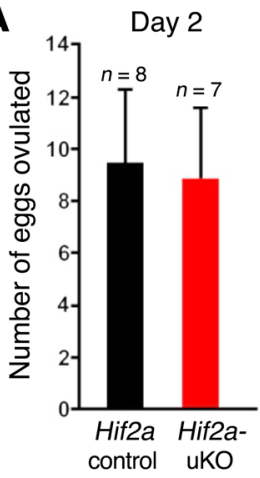

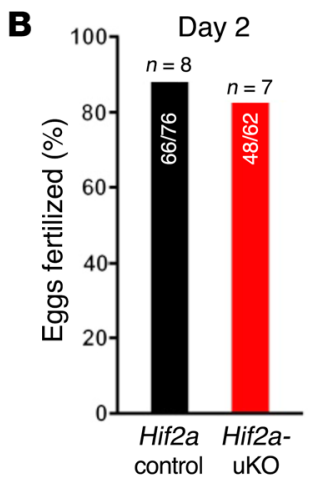

C

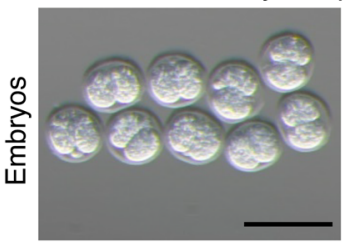

Hif2a control

$\mathbf{F}$
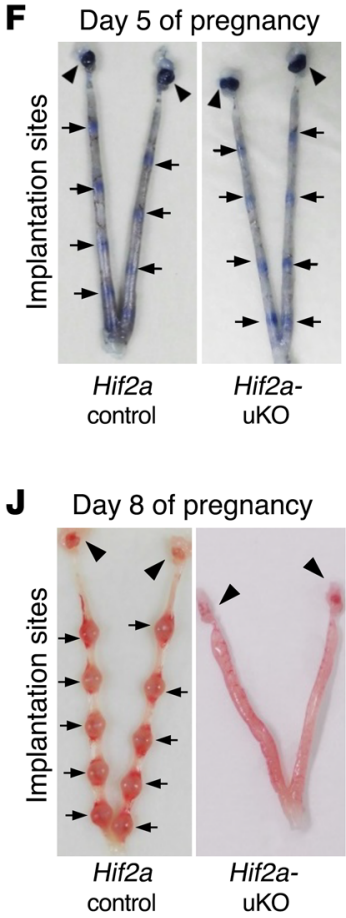
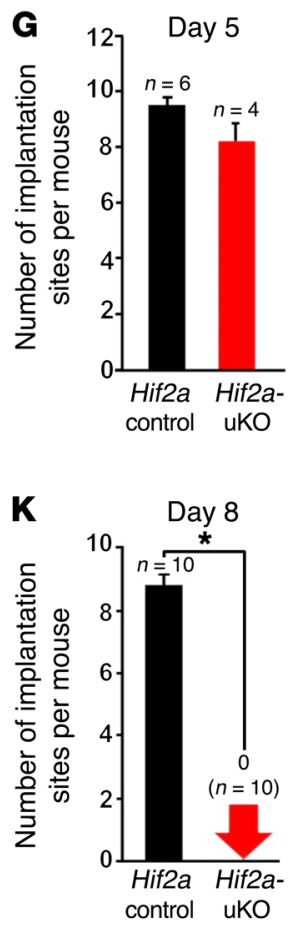

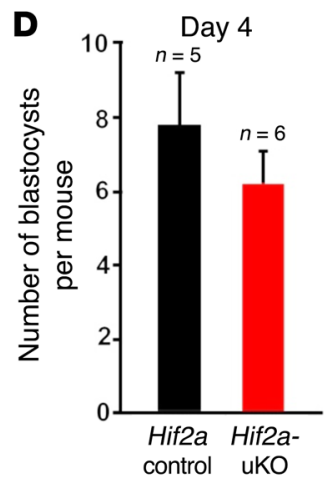

E

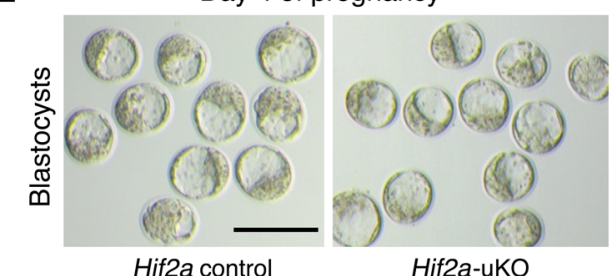

H
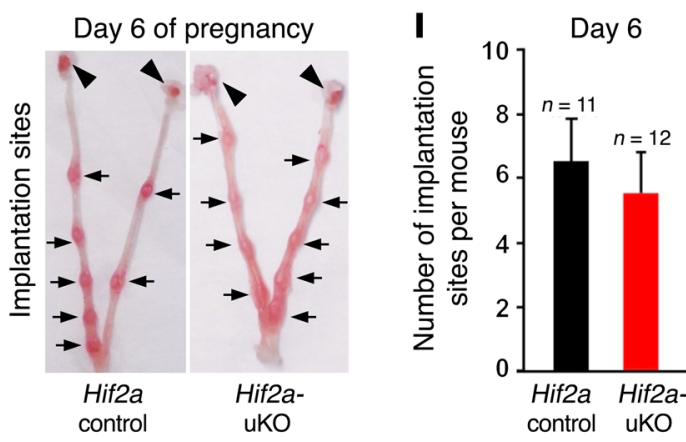

$\mathbf{L}$

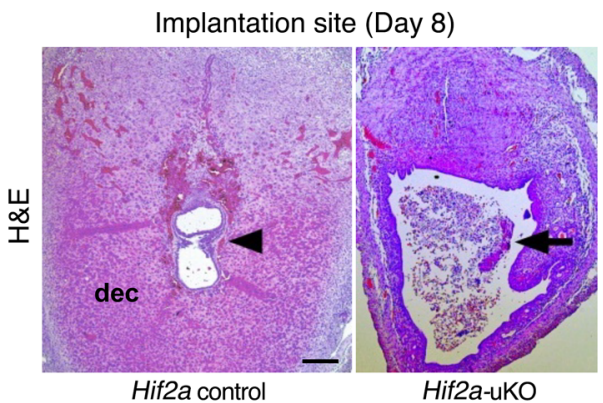

Figure 2. Hif2a-uKO mice show implantation failure. (A-E) Ovulation, fertilization, and development of preimplantation embryos were normal in Hif2auKO mice. A and D, $P>0.05$, mean \pm SEM, Student's $t$ test; $\mathbf{B}, P>0.05$, Fisher's exact probability test; $\mathbf{C}$ and $\mathbf{E}$, scale bar, $200 \mu \mathrm{m}$. (F and $\mathbf{G})$ Attachment reaction occurred normally in Hif2a-uKO mice at 1000 hours on day 5. $P>0.05$, mean \pm SEM, Student's $t$ test. Arrow and arrowhead indicate implantation site and ovary, respectively. (H and I) Decidualization was observed in Hif2a-uKO mice at 1000 hours on day 6. $P>0.05$, mean \pm SEM, Student's $t$ test. (J-L) Embryo implantation and decidualization were completely disturbed in Hif2a-uKO mice at 1000 hours on day 8 . In $\mathbf{K}$, ${ }^{*} P<0.05$, mean \pm SEM, Student's $t$ test. In L, arrowhead indicates an embryo; arrow, a destroyed embryo with blood cell infiltration; dec, decidua.

expected, serum $\mathrm{P}_{4}$ levels are significantly reduced in Hif $2 a$ uKO mice on days 6 and 8 of pregnancy when decidualization prominently occurs (Figure 3D). In contrast, serum estradiol-17 $\beta$ $\left(E_{2}\right)$ levels on days $4,5,6$, and 8 of pregnancy were comparable between controls and Hif2a-uKO mice (Figure 3E). To clarify whether the decreased levels of $\mathrm{P}_{4}$ were involved in decidual growth arrest of Hif2a-uKO mice, we examined the effect of luteal support in Hif $2 a$-uKO mice by daily subcutaneous injection of $\mathrm{P}_{4}$ from day 2 of pregnancy ( $2 \mathrm{mg} / \mathrm{mouse} /$ day). $\mathrm{P}_{4}$ administration successfully restored the number and weight of implantation sites in Hif2a-uKO mice on day 8 of pregnancy (Figure 3, F and G, and Supplemental Figure 3). The expression of decidualiza- 


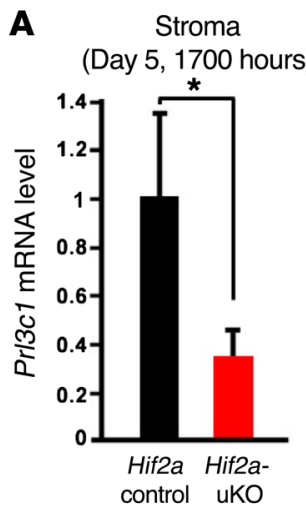

E

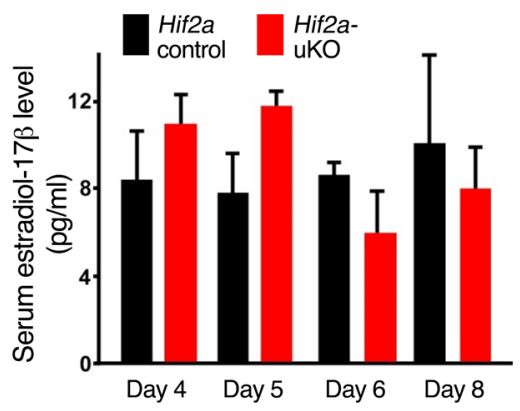

I

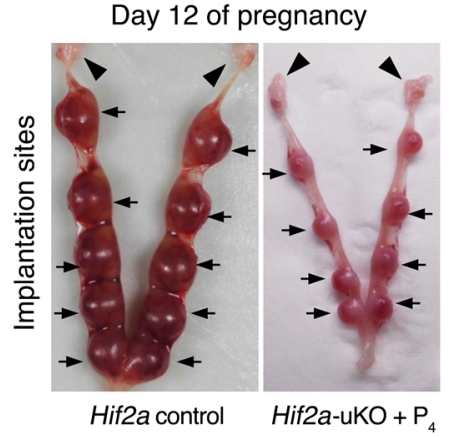

B

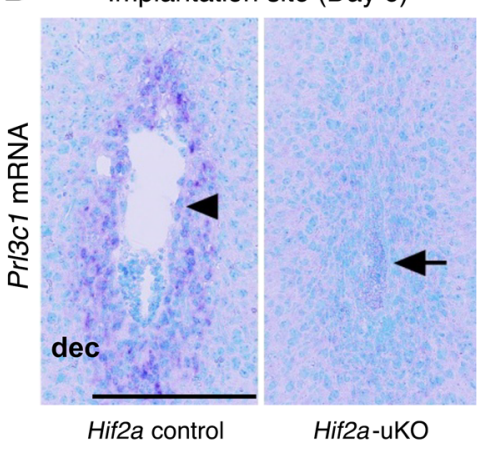

F

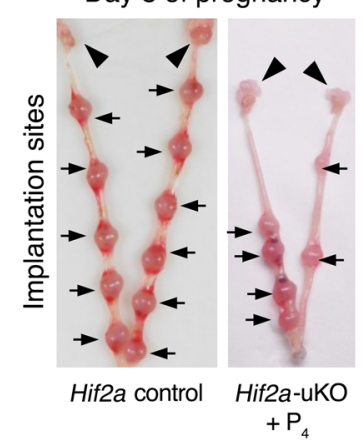

J

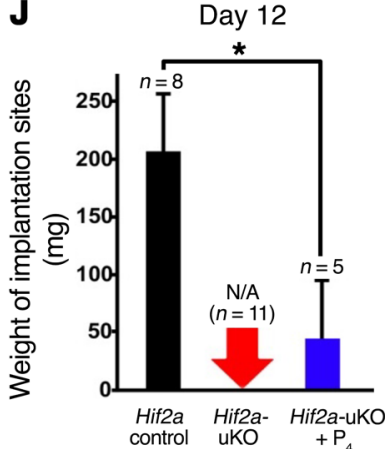

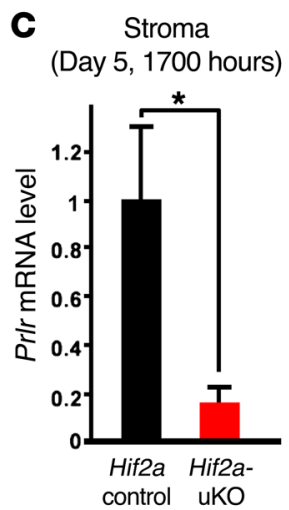

D

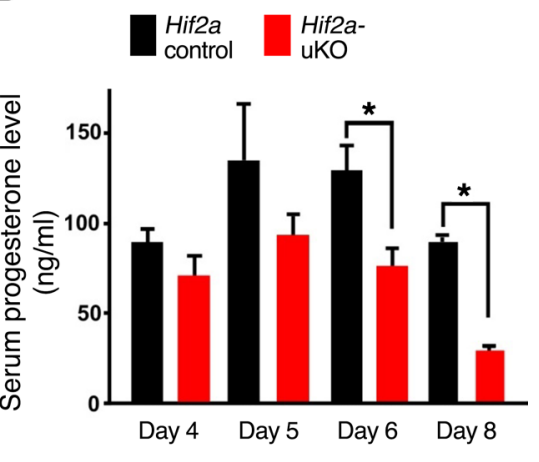

G Day 8

H Implantation site (Day 8)
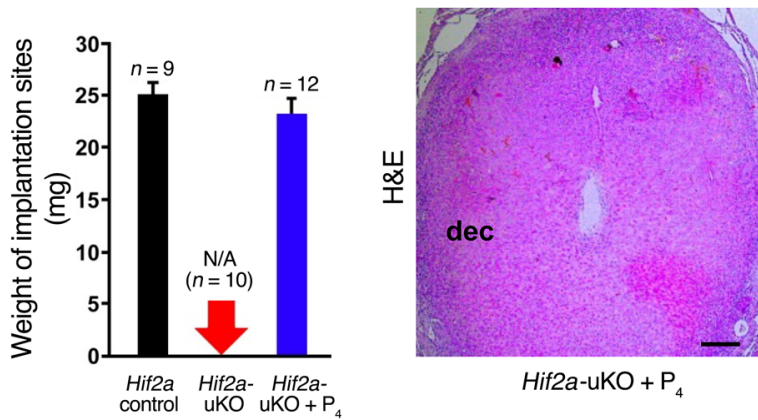

$\mathbf{K}$

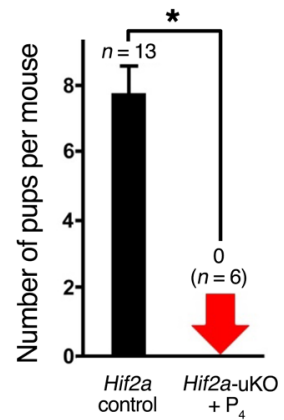

Figure 3. $\mathbf{P}_{\mathbf{4}}$ administration does not recover implantation failure but rescues decidualization in Hif2a-ukO mice. (A-C) Decidual prolactin-related factors Prl3c1 and Prlr, hallmarks of decidual health and luteotrophin, were reduced in Hif2a-uKO mice during decidualization. In $\mathbf{A}$ and $\mathbf{C}, n \geq 4,{ }^{*} P<0.05$, mean \pm SEM, Student's $t$ test. In B, scale bar, $200 \mu \mathrm{m}$; arrowhead, an embryo; arrow, a destroyed embryo with blood cell infiltration; dec, decidua. (D and $\mathbf{E})$ Serum $P_{4}$ levels were reduced in Hif2a-uKO mice compared with the controls on days 6 and 8 of pregnancy, whereas serum $\mathrm{E}_{2}$ levels were comparable between them. ${ }^{*} P<0.05$, mean $\pm S E M$, Student's $t$ test. (F and $\mathbf{C}$ ) Daily injection of $P_{4}$ from day 2 of pregnancy $(2 \mathrm{mg} / \mathrm{mouse} /$ day) restored decidualization in Hif2a-uKO mice on day 8 of pregnancy. $P>0.05$, mean \pm SEM, Student's $t$ test. Arrow and arrowhead indicate implantation site and ovary, respectively. $(\mathbf{H}) \mathrm{P}_{4}$ supplementation did not recover embryo survival in Hif2a-uKO mice on day 8 of pregnancy. No embryo was observed at the implantation site of Hif2a-uKO mice with $\mathrm{P}_{4}$ treatment. Scale bar, $200 \mu \mathrm{m}$; dec, decidua. (I-K) Hif2a-uKO mice with daily $\mathrm{P}_{4}$ injection showed the reduced weight of implantation sites on day 12 , and did not deliver any pups in parturition. ${ }^{*} P<0.05$, mean \pm SEM, Student's $t$ test.

tion marker BMP2 $(32,33)$ was comparable between Hif2a-uKO mice with $\mathrm{P}_{4}$ treatment and the control mice (Supplemental Figure 4), suggesting that $\mathrm{P}_{4}$ supplementation rescues decidualization failure in Hif $2 a$-uKO mice. However, H\&E staining demonstrated that $\mathrm{P}_{4}$ supplementation did not recover embryo survival in Hif2a-uKO mice on day 8 of pregnancy (Figure $3 \mathrm{H}$ ). These findings indicate that $\mathrm{P}_{4}$ reduction is not the cause of pregnancy failure in Hif $2 a$-uKO mice. In fact, Hif $2 a$-uKO mice with daily $\mathrm{P}_{4}$ injection showed reduced weight of implantation sites on day 12 (Figure 3, I and J) and never delivered pups (Figure 3K).
Pregnancy failure in Hif2a-uKO mice is due to insufficiency of uterine factors. $\mathrm{P}_{4}$ is a major ovarian factor essential for pregnancy, but the ovary produces other factors to support pregnancy. To evaluate the contribution of ovarian factors (including $\mathrm{P}_{4}$ ) to the implantation phenotype in Hif $2 a$-uKO mice, we performed transplantation of WT ovaries into Hif $2 a$-uKO mice or littermate controls by ovariectomy to normalize ovarian functions (Figure $4 \mathrm{~A}$ ) and evaluated the implantation phenotype of these mice. Normal implantation sites were observed in control mice with transplantation of WT ovaries on day 8 of pregnancy, while normal 
A

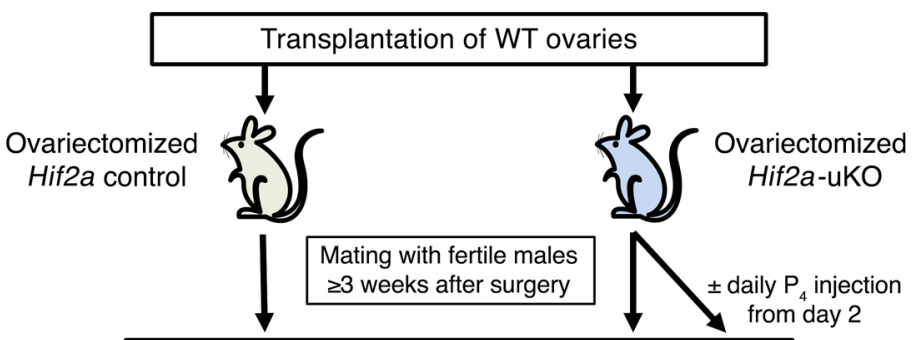

Evaluation of pregnancy phenotype on day 8

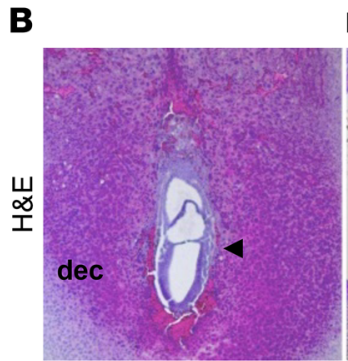

Hif2a control with WT ovaries
Implantation site (Day 8) c

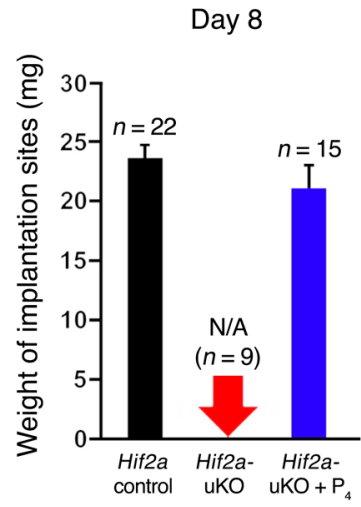

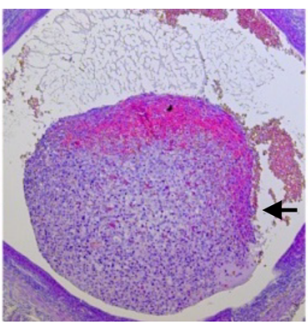

Hif2a-uKO with WT ovaries

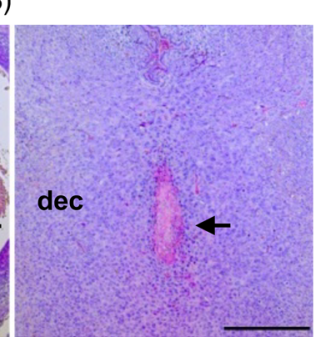

Hif2a-uKO with WT ovaries
D

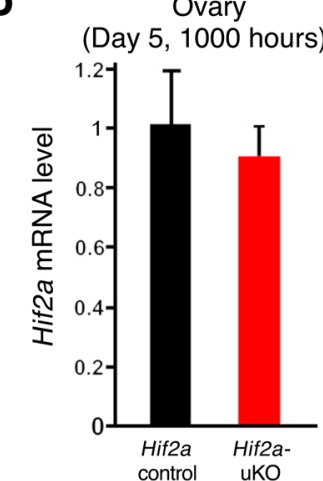

implantation sites were never observed in Hif2a-uKO mice that received WT ovaries (Figure 4, B and C). $\mathrm{P}_{4}$ supplementation did not recover implantation failure but did rescue decidualization in Hif 2a-uKO mice with ovarian transplantation (Figure 4, B and C). These experiments using mice with ovarian transplantation did not rescue the similar phenotypes observed in those without ovarian transplantation, indicating that implantation failure in Hif $2 a-$ $\mathrm{uKO}$ mice is not derived from ovarian factors but from a uterine factor. Importantly, ovarian Hif2a mRNA levels were comparable between Hif2a-uKO mice and their controls (Figure 4D).

Uterine HIF2 $\alpha$ confers appropriate homing of the implanting embryo in the crypt. To examine the detailed mechanism of implantation failure in Hif2a-uKO mice, we evaluated blastocyst attachment on day 5 morning. By histological analysis of Hif2a-deficient uteri, we found that the positioning of the embryo attachment at the bottom of the endometrial crypt was impaired in Hif2a-uKO mice (Figure 5A and Supplemental Figure 5). COX2, a marker of embryo attachment reaction (34), was similarly expressed in the implantation sites of both Hif2a-uKO and control mice (Figure 5, $B$ and C). The expression of LIF, a key regulator of embryo attachment $(9,10,35)$, was significantly decreased in Hif2a-deficient uteri on day 5 morning (Figure 5D). In accordance with LIF reduction,
Figure 4. Implantation failure in Hif2a-uKO mice is not triggered by ovarian factors but by uterine factors. (A) A scheme of transplantation of WT ovaries into Hif2a-uKO mice or the littermate controls with ovariectomy. (B and C) Ovarian transplantation of WT ovaries in Hif2a-uKO mice did not rescue the phenotype of implantation failure observed in those without ovarian transplantation. In B, scale bar, $500 \mu \mathrm{m}$; arrowhead, an embryo; arrow, a destroyed embryo with blood cell infiltration; dec, decidua. In C, $P>0.05$, mean \pm SEM, Student's $t$ test. (D) Ovarian Hif2a mRNA levels were comparable between Hif2a-uKO mice and their controls. $P>0.05, n=5$, mean \pm SEM, Student's $t$ test.

activation of STAT3, a downstream signaling effector of LIF (11), was eliminated in the implantation sites of Hif2a-uKO uteri, as evident from phosphorylated STAT3 immunostaining (Figure 5E). To evaluate whether the decrease of LIF in Hif2a-deficient uteri leads to implantation failure, we injected recombinant LIF (20 $\mu \mathrm{g} /$ mouse on day 4$)$ intraperitoneally in Hif2a-uKO mice in addition to $\mathrm{P}_{4}$ injection. Although LIF administration markedly normalized the positioning of embryo attachment to the crypt (Figure 5F and Supplemental Figure 5), it did not rescue pregnancy failure in Hif2a-uKO mice (Figure $5 \mathrm{G}$ ). Since LIF is transcriptionally regulated by $\operatorname{HIF}(36,37)$, uterine HIF $2 \alpha$ may determine the positioning of the embryo through LIF induction.

Persistent preservation of the intact luminal epithelium surrounding the embryo is associated with failed embryo invasion in Hif2a-uKO mice. We next used histological analysis to evaluate the process of embryo invasion from day 5 to day 6 in Hif2a-uKO mice. We found that alignment of the luminal epithelium disappeared at the implantation site and that trophoblast invasion starts in control mice at 2100 hours on day 5 (Figure 6A). In contrast, alignment of luminal epithelium was intact at the implantation site and trophoblast invasion did not occur in Hif2a-uKO mice at the same time (Figure 6A). On day 6 morning, blood cell infiltration without the normal embryo structure was observed at the implantation site of Hif2a-uKO mice, whereas the stromal area with trophoblast invasion expanded in the controls (Figure 6A). At 1700 hours on day 5 just before initiation of embryo invasion, epithelial expression of E-cadherin, a tight junction regulator as well as a marker of epithelium, was eliminated. Epithelial alignment collapsed at the implantation sites of the controls, whereas both E-cadherin expression and epithelial alignment persisted in Hif2a-uKO mice (Figure 6B), suggesting that intact alignment of luminal epithelium is associated with failed embryo invasion.

Direct contact between embryo and uterine stroma is involved in embryonic cell survival and invasion. Although the disappearance of the luminal epithelium attached to the embryo occurred in control mice on day 5 evening, apoptosis of the luminal epithelium was not observed at that time (Figure 7A), suggesting that apoptosis is not a key mechanism for the elimination of the luminal epithelium around the embryo. In contrast, apoptosis was observed in the trophoblast attached to the intact luminal epithelium in Hif2a-uKO mice, indicating programmed death in embryos that fail to initiate direct contact with endometrial stroma. We next hypothesized that epithelial mesenchymal transition (EMT) could be involved in the disappearance of the luminal epithelium around the embryo; 
A

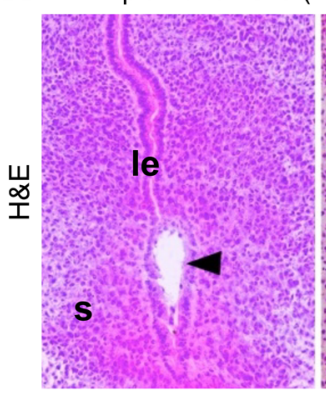

Hif2a control
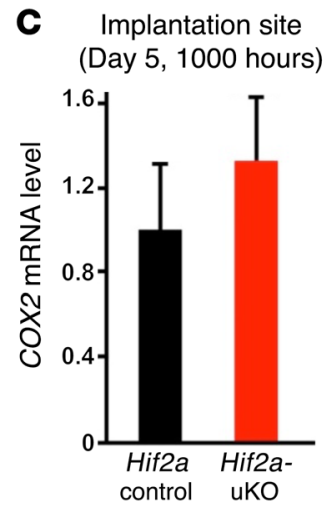

D Implantation site (Day 5, 1000 hours)

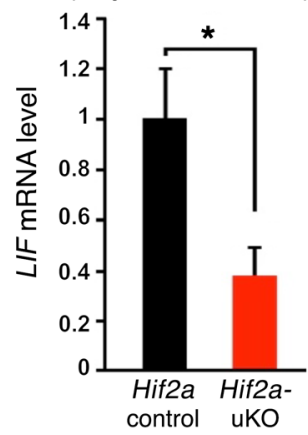

B

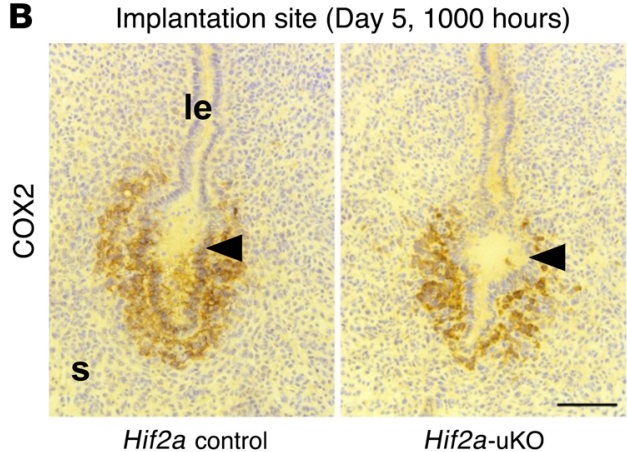

E Implantation site (Day 5, 1000 hours)

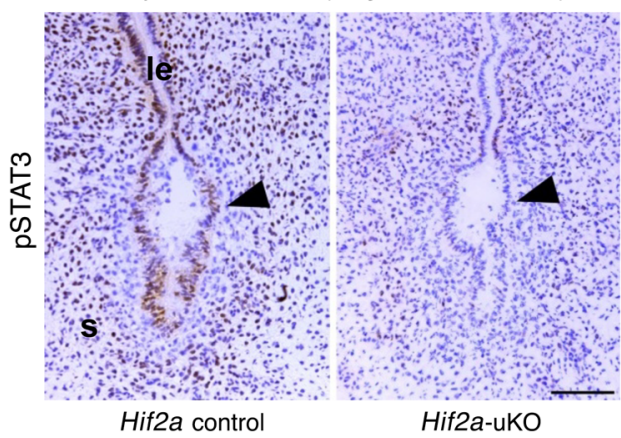

$\mathbf{F}$

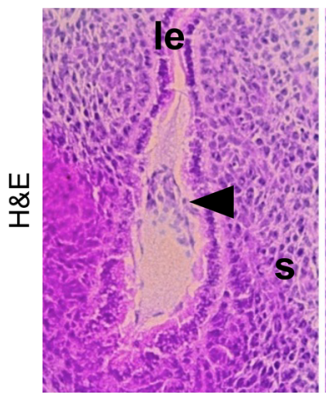

Hif2a control
Implantation site (Day 5, 1000 hours)

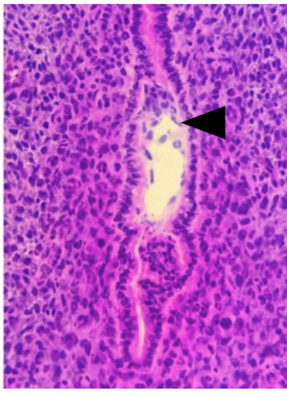

Hif2a-uKO

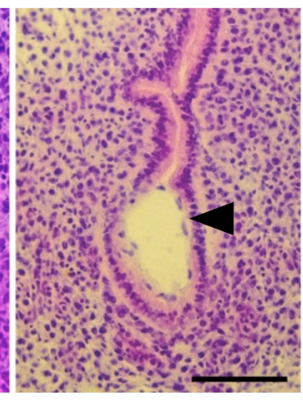

Hif2a-uKO $+\mathrm{P}_{4}+$ rLIF
G Implantation site (Day 6, 1000 hours)

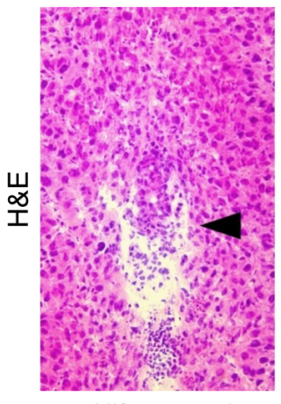

Hif2a control

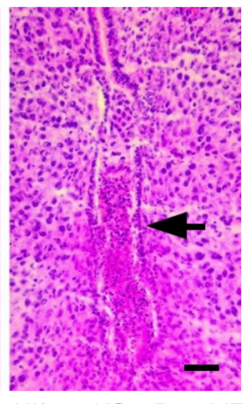

Hif2a-uKO $+\mathrm{P}_{4}+$ rLIF

Figure 5. Uterine HIF2 $\alpha$ places the implanting embryo in the bottom of the endometrial crypt through induction of LIF. (A) The positioning of embryo attachment at the bottom of the endometrial crypt was impaired in Hif2a-uKO mice at 1000 hours on day 5. Scale bar, $100 \mu \mathrm{m}$; arrowhead, an embryo; s, stroma; le, luminal epithelium. (B and C) COX2, a marker of embryo attachment reaction, was similarly expressed in the implantation sites of both Hif2auKO and control mice. $P>0.05, n \geq 5$, mean \pm SEM, Student's $t$ test. (D) The expression of LIF, a key regulator of embryo attachment, was decreased at the implantation site of Hif2a-uKO mice on day 5 morning. ${ }^{*} P<0.05, n \geq 5$, mean \pm SEM, Student's $t$ test. (E) Activation of STAT3, a downstream signaling of LIF, was eliminated in the implantation site of Hif2a-uKO mice, as demonstrated by phosphorylated STAT3 immunostaining. (F) Intraperitoneal injection of recombinant LIF ( $20 \mu \mathrm{g} /$ mouse on day 4 ) into Hif2a-uKO mice in addition to $\mathrm{P}_{4}$ injection normalized the position of embryo attachment to the bottom of the endometrial crypt on day 5 morning. (G) LIF administration could not rescue implantation failure in Hif2a-uKO mice on day 6 morning (arrow, a destroyed embryo with blood cell infiltration).

we investigated this process using R26GRR/Ltf-Cre mice, which can be used for tracing cells with uterine epithelium origin. However, epithelium-derived cells were not observed in the uterine stroma surrounding the invading embryo (Supplemental Figure 6), suggesting that EMT does not play a key role in the elimination of the luminal epithelium around the embryo.

Transmission electron microscopic analyses of embryo-uterine interface at 1900 hours on day 5 showed newly formed gaps between the stroma and the luminal epithelium and the invading trophoblast within these gaps in the control mice, but not in
Hif $2 a$-uKO mice (Figure 7B), suggesting a role for uterine HIF2 $\alpha$ in the detachment of the luminal epithelium from uterine stroma along with trophoblast invasion by direct contact with the stroma. Of note, this epithelial detachment was similarly observed in ICR mice on a different genetic background (Supplemental Figure 7), indicating that loss of the epithelial barrier is a common critical phenomenon during implantation.

In terms of the detachment of luminal epithelium from stroma, mRNA expression of membrane type 2 metalloproteinase (MT2-MMP) and lysyl oxidase (LOX) at the stroma of implan- 
A

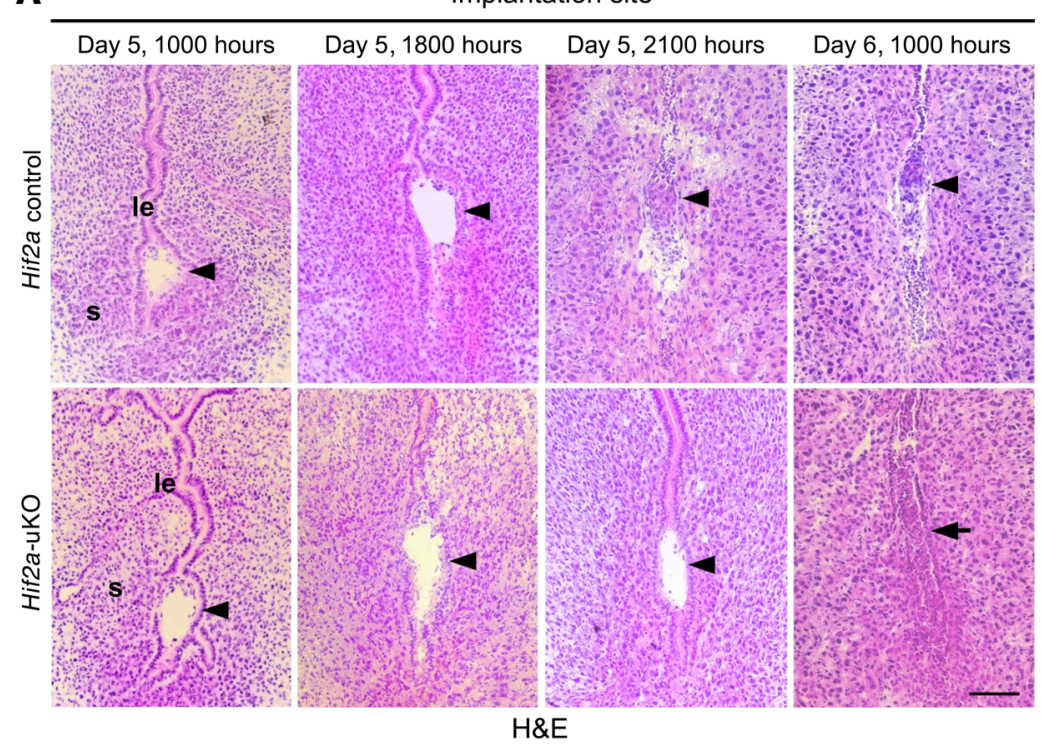

B

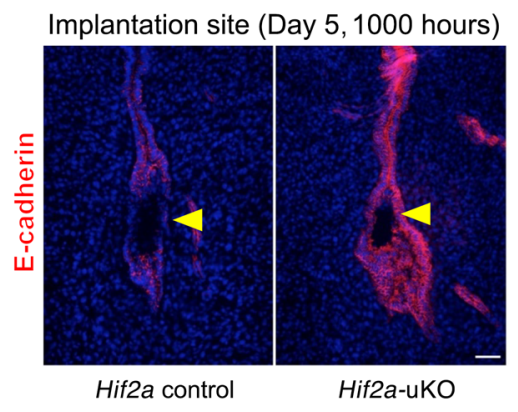

Figure 6. Intact alignment of luminal epithelium surrounding the embryo is associated with failed embryo invasion in Hif2a-uKO mice. (A) Alignment of luminal epithelium disappeared at the implantation site, and trophoblast invasion started in the control mice at 2100 hours on day 5 . In contrast, alignment of luminal epithelium was intact at the implantation site, and trophoblast invasion did not occur in Hif2a-uKO mice at the same time. On day 6 morning, destroyed embryo with blood cell infiltration was observed at the implantation site of Hif2a-uKO mice, while the stromal area with trophoblast invasion expanded in the controls. Scale bar, $200 \mu \mathrm{m}$; arrowhead, an embryo; s, stroma; le, luminal epithelium; arrow, a destroyed embryo with blood cell infiltration. (B) Expression of E-cadherin, a tight junction regulator as well as a marker of epithelium, was eliminated and epithelial alignment was collapsed at implantation sites of the controls, while both E-cadherin expression and epithelial alignment were persistent in Hif2a-uKO mice on day 5 evening.

tation sites collected by laser capture microdissection (LCM) was downregulated in Hif $2 a$-uKO mice compared with the controls (Figure 7, C and D). We also confirmed the downregulation of these genes by in situ hybridization (Supplemental Figures 8 and 9). MT2-MMP and LOX are known transcription targets of $\operatorname{HIF}(38,39)$. MT2-MMP, located on the cellular membrane, activates MMP2 outside the cell wall $(40,41)$ and breaks extracellular matrices composed of basement membrane together with the secreted MMP2, thereby serving as a regulator of basement membrane remodeling $(42,43)$. MT2-MMP also cleaves E-cadherin at the epithelial junction and regulates epithelial cell remodeling (44). LOX, secreted by the stroma, remodels the extracellular matrix by facilitating covalent cross-linking (45) and promotes cancer metastasis and invasion $(45,46)$. LOX and MT2-MMP in the stroma may cooperatively promote basement membrane remodeling and weaken the stroma-to-epithelium contact by changing the distribution of E-cadherin and the conformation of extracellular matrices between luminal epithelium and stroma. Our findings indicate that stromal Hif $2 a$ induces MMPs and LOX, which release the luminal epithelium from the stroma and promote trophoblast invasion.

Stromal Hif2a is critical for embryo invasion and activation of the PI3K-AKT pathway in the implanting embryo. To clarify the importance of stromal HIF $2 \alpha$ in embryo invasion, we generated mice with uterine stromal deletion of Hif $2 a$ (Hif $2 a$-sKO mice, Hif $2 a$-loxP/ Amhr2-Cre). We confirmed that Hif $2 a$ mRNA levels were significantly reduced in the stroma of Hif $2 a$-sKO mice (Supplemental Figure 10, A and B). As expected, the similar infertility phenotype with both impaired embryo invasion and persistent presence of luminal epithelium was observed in Hif2a-sKO mice (Figure 8, A-C), sug- gesting that stromal Hif 2 a controls embryo invasion. Moreover, we also generated mice with uterine epithelial deletion of Hif $2 a$ (Hif $2 a$ eKO mice, Hif $2 a$-loxP/Ltf-Cre) to evaluate the function of Hif $2 a$ in the luminal epithelium. Hif $2 a$ mRNA levels were significantly reduced in the luminal epithelium of Hif $2 a$-eKO mice (Supplemental Figure 10, C and D). However, Hif2a-eKO mice demonstrated normal fertility (Figure 8D), emphasizing the significance of stromal HIF2 $\alpha$. Taken together, these findings indicate that stromal HIF $2 \alpha$ detaches the luminal epithelium surrounding the embryo and activates an embryonic survival signal, which finally enables blastocyst invasion into the stroma.

In accordance with invasion capacity of the embryo, intensity of phosphorylated AKT staining was stronger in the control trophoblast compared with the Hif $2 a$-uKO trophoblast (Figure $8 \mathrm{E})$. FOXO1 is a transcriptional factor regulated by the PI3KAKT pathway (47). FOXO1 is phosphorylated by activated AKT and is translocated from the nucleus to the cytosol (47). Nuclear accumulation of FOXO1 was observed in the embryos attached to uteri of Hif $2 a$-uKO mice, although it was stained inside the cytosol in the invaded embryo of control mice (Supplemental Figure 11). These findings indicate that direct contact between the embryo and uterine stroma is required for activation of the PI3K-AKT-FOXO1 pathway as an embryonic survival signal to avoid programmed embryonic death. VEGF, adrenomedullin (ADM), and LOX, which can activate the PI3K-AKT pathway and are regulated by HIF (48-50), are expressed in the uterine stroma and involved in the process of embryo implantation (15, 51-54). We found that mRNA expression of VEGF and ADM as well as LOX at the stroma of implantation sites collected by LCM technique was significantly decreased in Hif $2 a$-uKO mice (Fig- 
A

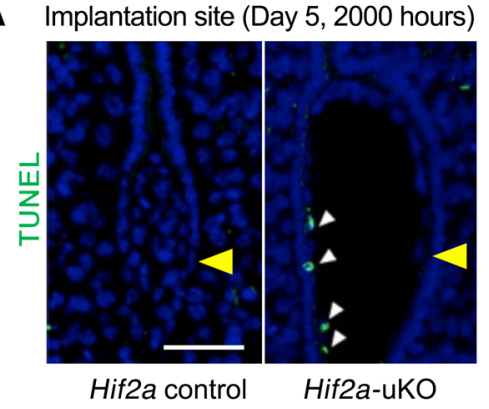

C

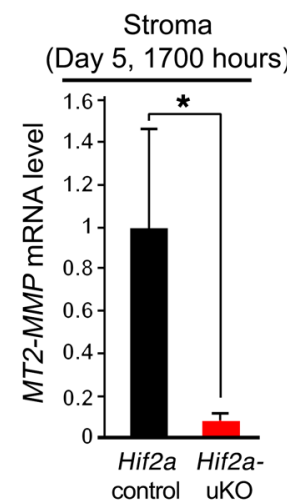

B
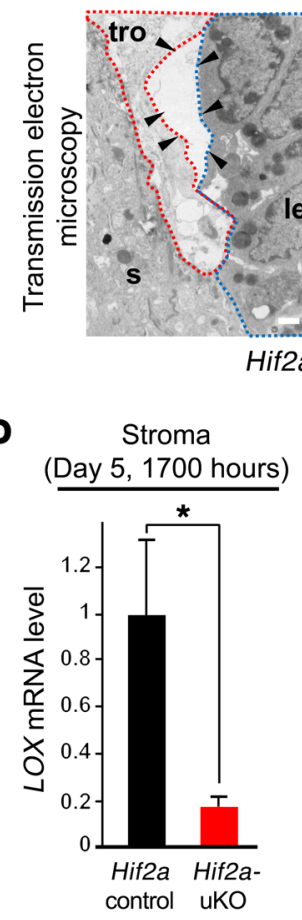

Implantation site (Day 5, 2000 hours)

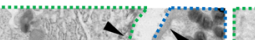

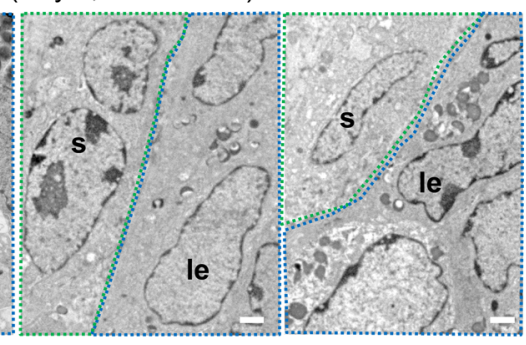

Hif2a-ukO
$\mathbf{S}$

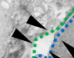

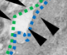
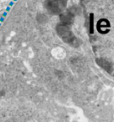

.

if2a control

Figure 7. Direct contact between embryo and uterine stroma is involved in embryonic cell survival and invasion. (A) Apoptosis of luminal epithelium was not apparently observed on day 5 evening, when the disappearance of luminal epithelium attached to the embryo occurred in the control mice. In contrast, apoptosis was observed in the trophoblast attached to intact luminal epithelium in Hif2a-uKO mice. Scale bar, $200 \mu \mathrm{m}$, arrowhead, an embryo. (B) Transmission electron microscopic analyses of embryo-uterine interface at 1900 hours on day 5 demonstrated the newly-formed gaps between the stroma and the luminal epithelium (arrowhead) and the invading trophoblast into these gaps in the control mice, but not in Hif2a-uKO mice. Scale bar, 1 $\mu \mathrm{m}$; s, stroma; tro, trophoblast; le, luminal epithelium. (C and $\mathbf{D})$ Expression of MT2-MMP and LOX at the stroma of implantation sites collected by laser capture microdissection was downregulated in Hif2a-uKO mice compared with the controls ${ }^{*} P<0.05, n=4$, mean \pm SEM, Student's $t$ test. ure 7D and Figure 8, F and G), suggesting that HIF2 $\alpha$-regulated stromal VEGF, ADM, and LOX activate the trophoblast cell survival pathway in normal conditions.

\section{Discussion}

In the present study, we found that mice with deletion of uterine Hif $2 a$ showed infertility because of pregnancy failure. Deficiency of uterine Hif2a downregulated the expression of decidual luteotrophin, which supports decidual growth through ovarian $\mathrm{P}_{4}$ production, but $\mathrm{P}_{4}$ administration did not recover implantation failure and rescued only decidualization. Mice with uterine deficiency of Hif $2 a$ displaced the implanting embryo in the aberrant position because of decreased LIF expression, but LIF supplementation normalized only the position of embryo attachment and did not recover implantation. These findings indicate that uterine HIF $2 \alpha$ contributes to successful implantation regardless of decidualization and the position of embryo attachment. More importantly, TEM revealed that the luminal epithelium attached to the embryo spontaneously detached from the stroma and that the embryo can directly contact the uterine stroma in control mice. Persistent alignment of the luminal epithelium surrounding the embryo with the inactivated embryonic survival signal blocked embryo invasion into the uterine stroma in Hif $2 a$-uKO mice (Figure 9). Direct contact between the embryo and the uterine stroma might be involved in embryonic cell survival and invasion in Hif $2 a-\mathrm{uKO}$ mice (Figure 9).

Since the surface of the endometrium is distant from uterine blood vessels, it is possible that oxygen concentration in the luminal epithelium and the stroma near embryos is relatively low compared with the inner endometrium and the myometrium (55). Therefore, it is estimated that the luminal epithelium before implantation is in a hypoxic state and that angiogenesis prominently occurs after implantation. After angiogenesis in implantation sites, oxygen concentration dynamically rises in the uterus. Previous studies have investigated the role of hypoxia in placental formation (56). On the other hand, few studies have focused on the role of hypoxia in the uterus during implantation. Hifla and Hif $2 a$ were shown to be strongly expressed in the mouse uterus during embryo implantation in a previous report (15), and HIF $\alpha$ is functionally critical for successful embryo implantation in our study. Physiological uterine hypoxia during implantation may contribute to embryo implantation through HIF, although factors other than hypoxia, such as embryonic stimuli, may be associated with the induction of HIF.

$\mathrm{P}_{4}$ signaling in the uterus is essential throughout pregnancy, and decidual luteotrophin activates the corpus luteum to maintain luteal function. Luteotrophin is one example of molecular interactions from the uterus to the ovary in rodents. In humans, chorionic gonadotrophin supports luteal function instead of decidual factors, suggesting that maintenance of luteal function by positive feedback of implantation-derived factors may be common machinery for successful pregnancy in mammals. The current study showed that luteal support cannot rescue implantation failure in mice with uterine deletion of Hif2a. These data are in accordance with the clinical fact that many patients do not become pregnant through IVF-ET treatment, even after sufficient luteal support. Dysregulation of HIF may be involved in recurrent implantation failure in humans.

Decreased LIF expression and aberrant position of the implantation site were observed in Hif $2 a$-uKO mice during embryo attachment. Since a previous report has demonstrated that HIF $2 \alpha$, not HIF1 $\alpha$, transcriptionally induces LIF expression in human colon cancer cell lines (37), it is possible that uterine HIF2 $\alpha$ induces LIF via transcriptional regulation in mice. In mouse embryonic stem cells, hypoxia-induced HIF1 $\alpha$ directly suppresses expression of the 
A

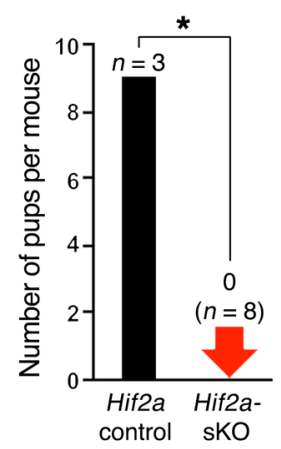

E

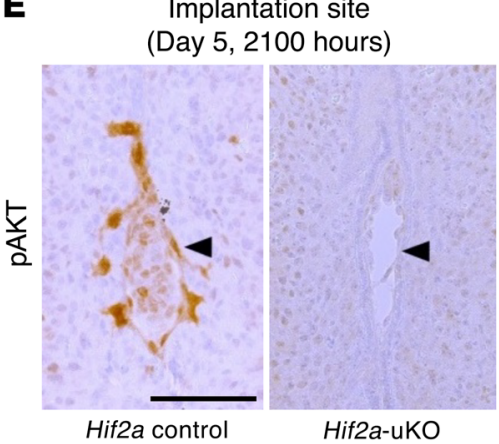

B

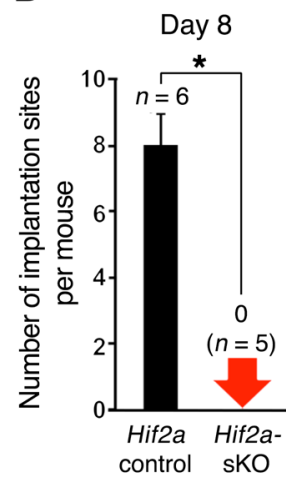

Hif2a-uKO
C

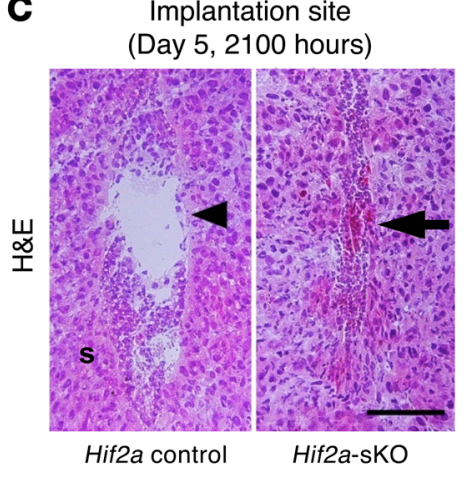

$\mathbf{F}$

Ftroma

(Day 5, 1700 hours)

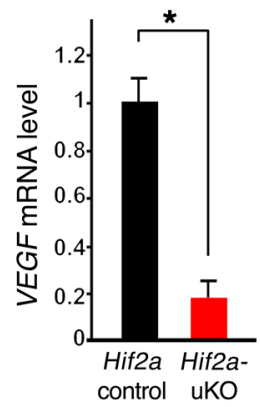

D

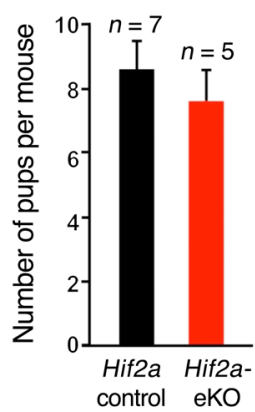

Stroma

(Day 5, 1700 hours)

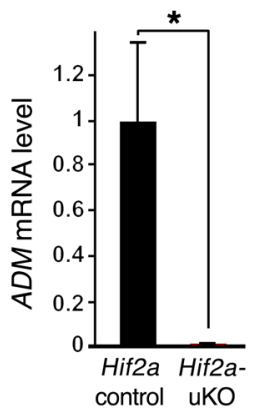

Figure 8. Stromal Hif2a is critical for embryo invasion and PI3K-AKT pathway is activated in the implanting embryos. (A-C) Infertility phenotype with both impaired embryo invasion and persistent presence of luminal epithelium was observed in Hif2a-sKO mice. In $\mathbf{A}$ and $\mathbf{B}$, ${ }^{*} P<0.05$, mean \pm SEM, Student's $t$ test. In C, scale bar, $200 \mu \mathrm{m}$; arrowhead, an embryo; s, stroma; arrow, a destroyed embryo with blood cell infiltration. (D) Hif2a-eKO mice demonstrated normal fertility. $P>0.05$, mean \pm SEM, Student's $t$ test. (E) Intensity of phosphorylated AKT staining was strong in the control trophoblast compared with Hif2a-uKO one. ( $F$ and $\mathbf{G}$ ) Expression of VEGF and ADM at the stroma of implantation sites collected by laser capture microdissection was decreased in Hif2a-uKO mice. ${ }^{*} P<0.05$, mean \pm SEM, Student's $t$ test.

LIF receptor through transcriptional regulation and inactivates LIFSTAT3 signaling (36). These findings indicate that HIF1 $\alpha$ and HIF2 $\alpha$ regulate LIF-STAT3 signaling in different and complex detail. As for the uterus, HIF2 $\alpha$ may dominantly activate LIF-STAT3 signaling.

Disappearance of the luminal epithelium might be an important process in embryo invasion. In addition to apoptosis, entosis and phagocytosis of the luminal epithelium by the trophoblast after attachment have been reported $(57,58)$. In control mice, apoptotic cells in the luminal epithelium at the implantation site are rarely observed; therefore, apoptosis as the cause behind the disappearance of the luminal epithelium can be ruled out. Similarly, entosis of the luminal epithelium was not observed in either Hif $2 a-\mathrm{uKO}$ or the control mice. Our observation demonstrated that EMT from the luminal epithelium to the stroma did not occur during implantation in mice. We discovered intermittent gaps between the luminal epithelium and the stroma that lead to the detachment of the luminal epithelium, which might be a critical process for embryo invasion; these processes are impaired in Hif $2 a-\mathrm{uKO}$ mice.

In concurrence with the detachment of the luminal epithelium from the stroma just before embryo invasion, the trophoblast enters the gap. Previous literature suggested the possibility that uterine stromal cells actively disrupt the epithelial basement membrane (59). The stroma produces several soluble factors, such as VEGF and ADM, to activate the PI3K-AKT-FOXO1 pathway as an embryonic survival signal and to promote trophoblast invasion into the stroma. MT2-MMP activates MMP2, breaks the extracellular matrix of basement membrane, and regulates epithelial E-cadherin distribution (40-42, 44). LOX debilitates the extracellular matrix of the basement membrane $(45,46)$. Therefore, LOX and MT2-MMP may cooperatively support remodeling of the basement membrane between the uterine stroma and the luminal epithelium. Since it remains unclear how the luminal epithelium detaches from the stroma and how embryonic activation occurs following invasion, further investigation is needed.

Dynamic molecular changes following embryo attachment are very complicated, and uterine HIF plays various roles in pregnancy. This study revealed that uterine HIF $2 \alpha$ is involved in a series of implantation processes, embryo attachment, decidualization, and especially, embryo invasion. The mechanism of embryo invasion has been scarcely reported, and we believe our results provide new evidence that stromal HIF $2 \alpha$ controls embryo invasion. The association between human implantation failure and HIF function needs to be clarified in future studies.

\section{Methods}

Mice. WT mice (C57BL/6J and ICR, Japan SLC), Hifla-floxed mice (16, 60), Hif2a-floxed mice $(60,61)$ (Jackson Laboratories), Ltf-Cre mice (21), Amhr2-Cre mice (19), Pgr-Cre mice (18), and Rosa26 Cre-reporter knock- 
A

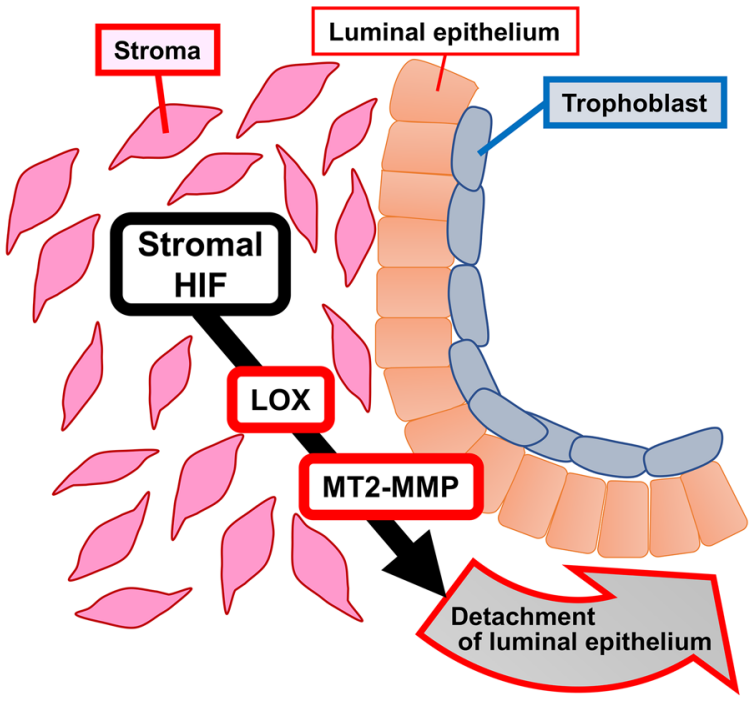

B

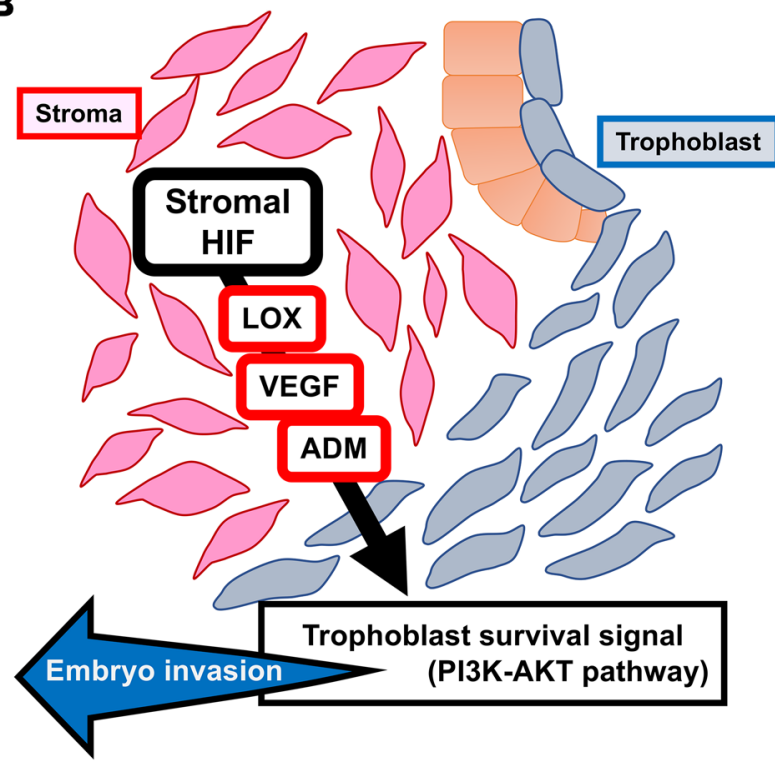

in mice (R26GRR mice) (62) were used in this study. R26GRR mice were provide by Ken-ichi Yagami (University of Tsukuba, Tsukuba, Japan) and RIKEN BRC (Tsukuba, Japan). Ltf-Cre mice were provided by Sudhansu K. Dey (Cincinnati Children's Hospital Medical Center, Cincinnati, OH, USA). Amhr2-Cre mice were provided by Richard Behringer (The University of Texas MD Anderson Cancer Center, Houston, TX, USA). Pgr-Cre mice were provided by Francesco J. DeMayo (National Institute of Environmental Health Sciences, Research Triangle Park, NC, USA). $L t f, A m h r 2$, and Pgr are expressed in the luminal epithelium, the stroma/ myometrium, and all the layers of uterus, respectively. Therefore, $L t f-$ Cre, Amhr2-Cre, and Pgr-Cre mice have the specific expression of Cre recombinase in the uterine epithelium, the stroma, and whole uterus, respectively (63). Mice with deletion of Hifla or Hif2a in whole uterus (Hifla-uKO mice and Hif2a-uKO mice, respectively) were generated by crossing Pgr-Cre with Hifla- or Hif2a-floxed mice. Mice with deletion of Hif2a in the uterine epithelium or in the stroma (Hif2a-eKO mice and Hif2a-sKO mice, respectively) were generated by crossing Hif2a-floxed mice with Ltf-Cre or Amhr2-Cre mice. Cre-negative homozygous littermates for the conditional alleles were used as controls. R26GRR mice
Figure 9. Potential pathways that contribute to embryo implantation through HIF. (A) Stromal HIF releases luminal epithelium from stroma through LOX and MT2-MMP, which permits direct contact between stroma and trophoblast. (B) Stromal HIF activates PI3K-AKT pathway as a trophoblast survival signal pathway through the induction of LOX, VEGF, and $A D M$, which promotes embryo invasion.

exhibit green emission before Cre-mediated recombination and red emission after the recombination. For epithelial cell lineage tracing in the uterus, we generated R26GRR/Ltf-Cre mice, which have red fluorescence in the uterine epithelium-derived cells (63).

Analysis of pregnancy events. For evaluation of pregnancy phenotypes, 2- to 5-month-old female mice of each line were mated with fertile males, and day 1 of pregnancy was defined as the day when we recognized vaginal plug. Ovulation, fertilization, preimplantation embryo development, and implantation were assessed as described (64). To treat Hif $2 a$-uKO mice with exogenous $\mathrm{P}_{4}$ and recombinant LIF protein, daily subcutaneous injections of $\mathrm{P}_{4}(2 \mathrm{mg} / \mathrm{mouse} /$ day $)$ from day 2 of pregnancy and intraperitoneal injection of recombinant LIF (20 $\mu \mathrm{g} /$ mouse) at 0900 hours on day 4 of pregnancy were performed, respectively. Recombinant LIF was produced using LIF expression vector as previously described $(65,66)$. The LIF expression vector was provided by Eiichi Hondo (Nagoya University, Japan).

Ovarian transplantation. Ovarian transplantation was performed as described (67). Female mice were anesthetized, the ovarian fat pad with the ovary was exteriorized, the bursa opened, and ovaries removed. The excised ovaries were held briefly in M2 medium and grafted back into the bursal cavity of recipient mice. More than 3 weeks after transplantation, the mice with ovary transplantation were mated with fertile WT males.

$H \& E$ staining and immunostaining. Frozen sections $(12 \mu \mathrm{m})$, or formalin-fixed paraffin-embedded sections $(6 \mu \mathrm{m})$ were subjected to H\&E staining and immunohistochemistry. For immunostaining, antibodies against Ki67 (SP6, Thermo Fisher Scientific), and phosphorylated STAT3 (pSTAT3; EP2147Y, Abcam), COX2 (AA570-598, Cayman Chemical), E-cadherin (24E10, Cell Signaling Technology), phosphorylated AKT (pAKT; D9E, Cell Signaling Technology), and FOXO1 (C29H4, Cell Signaling Technology) were used.

Fluorescence assay. Frozen sections $(12 \mu \mathrm{m})$ were subjected to immunofluorescence. Alexa Fluor 488-conjugated anti-rat immunoglobulin G and Alexa Fluor 555-conjugated anti-rabbit immunoglobulin $\mathrm{G}$ were used for protein detection, and nuclear staining was performed with 4, 6-diamidino-2-phenylindole using a fluorescence microscope (DM5000B and HyD, Leica Microsystems GmbH).

Measurement of serum $\mathrm{P}_{4}$ and $\mathrm{E}_{2}$ levels. Blood samples from mice were collected on the indicated days of pregnancy. Serum was separated by centrifugation ( $850 \mathrm{~g}$ for 15 minutes) and stored at $-80^{\circ} \mathrm{C}$ until analysis. Serum $\mathrm{P}_{4}$ levels were measured by Progesterone EIA kit (Cayman Chem$\mathrm{ical}$ ), and $\mathrm{E}_{2}$ levels were measured by Estradiol ELISA kit (CalBiotech).

In situ hybridization. Sense or antisense DIG-labeled cRNA probes for Prl3c1, MT2-MMP, and LOX were generated using appropriate polymerases from respective cDNAs as previously described (68). Specific RNA probes were prepared by DIG Labeling Kit (Roche Diagnostics $\mathrm{GmbH}$ ) and paraffin sections $(6 \mu \mathrm{m})$ were incubated with the probes and colored using NBT/BCIP solution (Roche Diagnostics $\mathrm{GmbH}$ ) counterstained by methyl green solution (Wako Pure Chemical Industries). 
Laser capture microdissection. LCM was performed as previously described (7). Frozen sections $(20 \mu \mathrm{m})$ were mounted on polyethylene naphthalate slides (Leica Microsystems), fixed in cold acetone, stained in $0.05 \%$ toluidine blue, and dehydrated in ethanol. The target regions in the sections were microdissected with a LMD7000 system (Leica Microsystems). The luminal epithelium and stroma close to a crypt at the antimesometrial pole were dissected out from the uteri by LCM.

RT-quantitative PCR ( $q P C R)$. For conventional RNA analyses, total RNA extraction from frozen whole tissues was performed as previously described (7). As for LCM samples, the extracted RNA was amplified using an Ovation PicoSL WTA system V2 (NuGEN Technology), and qPCR was performed as previously described (7). A housekeeping gene ( $\beta$-actin $[$ Actb]) was used as an internal standard for normalizing the relative mRNA expression. Sequences of $\mathrm{qPCR}$ primers are listed in Supplemental Table 1.

TUNEL assay. In situ detection of apoptosis was performed using the In Situ Cell Death Detection Kit (Roche) following the manufacturer's instructions. The signal was detected using a confocal laser scanning microscope (LSM700, Carl Zeiss Microscopy GmbH).

Transmission electron microscopy. Uterine tissues were fixed with a buffered mix of $2.5 \%$ glutaraldehyde and $1.6 \%$ paraformaldehyde in phosphate buffer (0.1 M, pH 7.4) for 17 hours, and postfixed with 0.1 $\mathrm{M}$ cacodylate buffer and 1\% osmium tetroxide for 30 minutes, dehydrated, replaced with propylene oxide, embedded in epoxy resin, and hardened at $60^{\circ} \mathrm{C}$ for 24 hours. The tissues were sectioned using a diamond knife at a thickness of 60-70 nm, mounted onto single slot copper grids with a support film, stained with lead citrate and uranyl acetate, and imaged at $80 \mathrm{kV}$ in a transmission electron microscope (JEM-1011, JEOL).

Statistics. Statistical analyses were performed using a 2-tailed Student's $t$ test and Fisher's exact probability test. A value of $P<0.05$ was considered statistically significant.

Study approval. All mice used in this investigation were housed in the University of Tokyo Animal Care Facility according to the institutional guidelines for the use of laboratory animals. All the animal experi- ments in the present study were reviewed and approved by the animal experiment committee of the University of Tokyo (Tokyo, Japan).

\section{Author contributions}

YH and TSF designed the study. LM, YH, TSF, TT, SA, TH, and SI performed experiments and collected the data. NT, HF, RSH, $\mathrm{MM}, \mathrm{HH}, \mathrm{MSK}$, and TF provided technical assistance for the experiments. LM, YH, and TSF analyzed the data. YH, TSF, NT, $\mathrm{RSH}$, and YO discussed and interpreted the results. YH and TSF wrote the manuscript. $\mathrm{RSH}$ critically reviewed the manuscript. YH supervised the study.

\section{Acknowledgments}

We thank Ken-ichi Yagami (University of Tsukuba) and RIKEN BRC through the National Bio-Resource Project of the MEXT, Japan, for providing R26GRR mice, Sudhansu K. Dey (Cincinnati Children's Hospital Medical Center) for providing Ltf-Cre mice and reviewing the manuscript critically, Richard Behringer (The University of Texas MD Anderson Cancer Center) for providing Amhr2-Cre mice, Francesco J. DeMayo (National Institute of Environmental Health Sciences) for providing Pgr-Cre mice, Eiichi Hondo (Nagoya University) for providing the LIF expression vector, and Katie A. Gerhardt (Cincinnati Children's Hospital Medical Center) for efficient editing of the manuscript. This work was supported by grants from the Japan Agency for Medical Research and Development (AMED) PRIME (JP18gm5910010), the Japan Society for the Promotion of Science, KAKENHI (16H04679, 16K15701, 16K15700, 16H05469, 15K10660, $15 \mathrm{H} 04979,17 \mathrm{~K} 16833,17 \mathrm{H} 06640,15 \mathrm{~K} 15596,16 \mathrm{~K} 10668)$, and the Takeda Science Foundation.

Address correspondence to: Yasushi Hirota, Department of Obstetrics and Gynecology, Graduate School of Medicine, The University of Tokyo, 7-3-1 Hongo, Bunkyo-ku, Tokyo 113-8655, Japan. Phone: 81.3.3815.5411; Email: yhirota-tky@umin.ac.jp.
1. Inhorn MC, Patrizio P. Infertility around the globe: new thinking on gender, reproductive technologies and global movements in the 21st century. Hum Reprod Update. 2015;21(4):411-426.

2. Dey SK, et al. Molecular cues to implantation. Endocr Rev. 2004;25(3):341-373.

3. Cha J, Sun X, Dey SK. Mechanisms of implantation: strategies for successful pregnancy. Nat Med. 2012;18(12):1754-1767.

4. Egashira M, Hirota Y. Uterine receptivity and embryo-uterine interactions in embryo implantation: lessons from mice. Reprod Med Biol. 2013;12(4):127-132.

5. Matsumoto H, Fukui E, Yoshizawa M. Molecular and cellular events involved in the completion of blastocyst implantation. Reprod Med Biol. 2016;15(2):53-58.

6. Tranguch S, Wang H, Daikoku T, Xie H, Smith DF, Dey SK. FKBP52 deficiency-conferred uterine progesterone resistance is genetic background and pregnancy stage specific. J Clin Invest. 2007;117(7):1824-1834.

7. Haraguchi H, et al. MicroRNA-200a locally attenuates progesterone signaling in the cervix, preventing embryo implantation. Mol Endocrinol. 2014;28(7):1108-1117.

8. Das SK, et al. Heparin-binding EGF-like growth factor gene is induced in the mouse uterus temporally by the blastocyst solely at the site of its apposition: a possible ligand for interaction with blastocyst EGF-receptor in implantation. Development. 1994;120(5):1071-1083.

9. Chen JR, Cheng JG, Shatzer T, Sewell L, Hernandez L, Stewart CL. Leukemia inhibitory factor can substitute for nidatory estrogen and is essential to inducing a receptive uterus for implantation but is not essential for subsequent embryogenesis. Endocrinology. 2000;141(12):4365-4372.

10. Song H, Lim H, Das SK, Paria BC, Dey SK. Dysregulation of EGF family of growth factors and COX-2 in the uterus during the preattachment and attachment reactions of the blastocyst with the luminal epithelium correlates with implantation failure in LIF-deficient mice. Mol Endocrinol. 2000;14(8):1147-1161.

11. Cheng JG, Chen JR, Hernandez L, Alvord WG, Stewart CL. Dual control of LIF expression and LIF receptor function regulate Stat 3 activation at the onset of uterine receptivity and embryo implantation. Proc Natl Acad Sci U S A. 2001;98(15):8680-8685.

12. Dengler VL, Galbraith M, Espinosa JM. Transcriptional regulation by hypoxia inducible factors. Crit Rev Biochem Mol Biol. 2014;49(1):1-15.

13. Schödel J, Oikonomopoulos S, Ragoussis J, Pugh CW, Ratcliffe PJ, Mole DR. High-resolution genome-wide mapping of HIF-binding sites by ChIP-seq. Blood. 2011;117(23):e207-e217.

14. Majmundar AJ, Wong WJ, Simon MC. Hypoxiainducible factors and the response to hypoxic stress. Mol Cell. 2010;40(2):294-309.

15. Daikoku T, et al. Expression of hypoxia-inducible factors in the peri-implantation mouse uterus is regulated in a cell-specific and ovarian steroid hormone-dependent manner. Evidence for differential function of HIFs during early pregnancy. J Biol Chem. 2003;278(9):7683-7691.

16. Ryan HE, Lo J, Johnson RS. HIF-1 alpha is required for solid tumor formation and embryonic vascularization. EMBO J. 1998;17(11):3005-3015.

17. Tian H, Hammer RE, Matsumoto AM, Russell 
DW, McKnight SL. The hypoxia-responsive transcription factor EPAS1 is essential for catecholamine homeostasis and protection against heart failure during embryonic development. Genes Dev. 1998;12(21):3320-3324.

18. Soyal SM, et al. Cre-mediated recombination in cell lineages that express the progesterone receptor. Genesis. 2005;41(2):58-66.

19. Jamin SP, Arango NA, Mishina Y, Hanks MC, Behringer RR. Requirement of Bmprla for Müllerian duct regression during male sexual development. Nat Genet. 2002;32(3):408-410.

20. Daikoku T, Jackson L, Besnard V, Whitsett J, Ellenson LH, Dey SK. Cell-specific conditional deletion of Pten in the uterus results in differential phenotypes. Gynecol Oncol. 2011;122(2):424-429.

21. Daikoku T, Ogawa Y, Terakawa J, Ogawa A, DeFalco T, Dey SK. Lactoferrin-iCre: a new mouse line to study uterine epithelial gene function. Endocrinology. 2014;155(7):2718-2724.

22. Herz Z, Khan I, Jayatilak PG, Gibori G. Evidence for the secretion of decidual luteotropin: a prolactin-like hormone produced by rat decidual cells. Endocrinology. 1986;118(6):2203-2209.

23. Bao L, et al. Decidual prolactin silences the expression of genes detrimental to pregnancy. Endocrinology. 2007;148(5):2326-2334.

24. Jayatilak PG, Glaser LA, Basuray R, Kelly PA, Gibori G. Identification and partial characterization of a prolactin-like hormone produced by rat decidual tissue. Proc Natl Acad Sci U S A. 1985;82(1):217-221.

25. Cha J, et al. Combinatory approaches prevent preterm birth profoundly exacerbated by gene-environment interactions. J Clin Invest. 2013;123(9):4063-4075.

26. Binart N, et al. Rescue of preimplantatory egg development and embryo implantation in prolactin receptor-deficient mice after progesterone administration. Endocrinology. 2000;141(7):2691-2697.

27. Reese J, et al. Implantation and decidualization defects in prolactin receptor (PRLR)-deficient mice are mediated by ovarian but not uterine PRLR. Endocrinology. 2000;141(5):1872-1881.

28. Alam SM, Konno T, Sahgal N, Lu L, Soares MJ. Decidual cells produce a heparin-binding prolactin family cytokine with putative intrauterine regulatory actions. J Biol Chem. 2008;283(27):18957-18968.

29. Hiraoka Y, et al. PLP-I: a novel prolactinlike gene in rodents. Biochim Biophys Acta. 1999;1447(2-3):291-297.

30. Toft DJ, Linzer DI. Prolactin (PRL)-like protein J, a novel member of the PRL/growth hormone family, is exclusively expressed in maternal decidua. Endocrinology. 1999;140(11):5095-5101.

31. Ishibashi K, Imai M. Identification of four new members of the rat prolactin/growth hormone gene family. Biochem Biophys Res Commun. 1999;262(3):575-578.

32. Paria BC, et al. Cellular and molecular responses of the uterus to embryo implantation can be elicited by locally applied growth factors. Proc Natl Acad Sci U S A. 2001;98(3):1047-1052.

33. Lee KY, et al. Bmp2 is critical for the murine uterine decidual response. Mol Cell Biol.
2007;27(15):5468-5478.

34. $\operatorname{Lim} \mathrm{H}$, et al. Multiple female reproductive failures in cyclooxygenase 2-deficient mice. Cell. 1997;91(2):197-208.

35. Stewart CL, et al. Blastocyst implantation depends on maternal expression of leukaemia inhibitory factor. Nature. 1992;359(6390):76-79.

36. Jeong $\mathrm{CH}$, et al. Hypoxia-inducible factor-1 alpha inhibits self-renewal of mouse embryonic stem cells in Vitro via negative regulation of the leukemia inhibitory factor-STAT3 pathway. J Biol Chem. 2007;282(18):13672-13679.

37. Wu L, et al. HIF-2 $\alpha$ mediates hypoxia-induced LIF expression in human colorectal cancer cells. Oncotarget. 2015;6(6):4406-4417.

38. Zhu S, et al. Transcriptional upregulation of MT2-MMP in response to hypoxia is promoted by HIF-1 $\alpha$ in cancer cells. Mol Carcinog. 2011;50(10):770-780.

39. Erler JT, et al. Lysyl oxidase is essential for hypoxia-induced metastasis. Nature. 2006;440(7088):1222-1226.

40. Sato H, Seiki M. Membrane-type matrix metalloproteinases (MT-MMPs) in tumor metastasis. J Biochem. 1996;119(2):209-215.

41. Morrison CJ, Overall CM. TIMP independence of matrix metalloproteinase (MMP)-2 activation by membrane type 2 (MT2)-MMP is determined by contributions of both the MT2-MMP catalytic and hemopexin C domains. J Biol Chem. 2006;281(36):26528-26539.

42. Hotary K, Li XY, Allen E, Stevens SL, Weiss SJ. A cancer cell metalloprotease triad regulates the basement membrane transmigration program. Genes Dev. 2006;20(19):2673-2686.

43. Ota I, Li XY, Hu Y, Weiss SJ. Induction of a MT1-MMP and MT2-MMP-dependent basement membrane transmigration program in cancer cells by Snail1. Proc Natl Acad Sci U S A. 2009;106(48):20318-20323.

44. Gómez-Escudero J, et al. E-cadherin cleavage by MT2-MMP regulates apical junctional signaling and epithelial homeostasis in the intestine. J Cell Sci. 2017;130(23):4013-4027.

45. Cox TR, Gartland A, Erler JT. Lysyl oxidase, a targetable secreted molecule involved in cancer metastasis. Cancer Res. 2016;76(2):188-192.

46. Erler JT, et al. Hypoxia-induced lysyl oxidase is a critical mediator of bone marrow cell recruitment to form the premetastatic niche. Cancer Cell. 2009;15(1):35-44.

47. Gilley J, Coffer PJ, Ham J. FOXO transcription factors directly activate bim gene expression and promote apoptosis in sympathetic neurons. J Cell Biol. 2003;162(4):613-622.

48. Jiang BH, Liu LZ. PI3K/PTEN signaling in tumorigenesis and angiogenesis. Biochim Biophys Acta. 2008;1784(1):150-158.

49. Nagaya N, Mori H, Murakami S, Kangawa K, Kitamura S. Adrenomedullin: angiogenesis and gene therapy. Am J Physiol Regul Integr Comp Physiol. 2005;288(6):R1432-R1437.

50. Pez F, et al. The HIF-1-inducible lysyl oxidase activates HIF-1 via the Akt pathway in a positive regulation loop and synergizes with HIF-1 in promoting tumor cell growth. Cancer Res. 2011;71(5):1647-1657.
51. Chakraborty I, Das SK, Dey SK. Differential expression of vascular endothelial growth factor and its receptor mRNAs in the mouse uterus around the time of implantation.J Endocrinol. 1995;147(2):339-352.

52. Li M, Yee D, Magnuson TR, Smithies O, Caron KM. Reduced maternal expression of adrenomedullin disrupts fertility, placentation, and fetal growth in mice. JClin Invest. 2006;116(10):2653-2662.

53. Li M, Wu Y, Caron KM. Haploinsufficiency for adrenomedullin reduces pinopodes and diminishes uterine receptivity in mice. Biol Reprod. 2008;79(6):1169-1175.

54. Li SY, et al. Molecular characterization of lysyl oxidase-mediated extracellular matrix remodeling during mouse decidualization. FEBS Lett. 2017;591(10):1394-1407.

55. Rodesch F, Simon P, Donner C, Jauniaux E. Oxygen measurements in endometrial and trophoblastic tissues during early pregnancy. Obstet Gynecol.1992;80(2):283-285.

56. Aplin JD. Hypoxia and human placental development. JClin Invest. 2000;105(5):559-560.

57. Li Y, Sun X, Dey SK. Entosis allows timely elimination of the luminal epithelial barrier for embryo implantation. Cell Rep. 2015;11(3):358-365.

58. Aplin JD, Ruane PT. Embryo-epithelium interactions during implantation at a glance. J Cell Sci. 2017;130(1):15-22.

59. Schlafke S, Enders AC. Cellular basis of interaction between trophoblast and uterus at implantation. Biol Reprod. 1975;12(1):41-65.

60. Semba H, et al. HIF-1 $\alpha$-PDK1 axis-induced active glycolysis plays an essential role in macrophage migratory capacity. Nat Commun. 2016;7:11635.

61. Gruber M, Hu CJ, Johnson RS, Brown EJ, Keith B, Simon MC. Acute postnatal ablation of Hif-2alpha results in anemia. Proc Natl Acad Sci US A. 2007;104(7):2301-2306.

62. Hasegawa Y, et al. Novel ROSA26 Cre-reporter knock-in C57BL/6N mice exhibiting green emission before and red emission after Cre-mediated recombination. Exp Anim. 2013;62(4):295-304 .

63. Hiraoka T, et al. STAT3 accelerates uterine epithelial regeneration in a mouse model of decellularized uterine matrix transplantation. JCI Insight. 2016;1(8):e87591.

64. Hirota Y, Daikoku T, Tranguch S, Xie H, Bradshaw HB, Dey SK. Uterine-specific p53 deficiency confers premature uterine senescence and promotes preterm birth in mice. JClin Invest. 2010;120(3):803-815.

65. Terakawa J, et al. Embryo implantation is blocked by intraperitoneal injection with anti-LIF antibody in mice. J Reprod Dev. 2011;57(6):700-707.

66. Kobayashi R, et al. The contribution of leukemia inhibitory factor (LIF) for embryo implantation differs among strains of mice. Immunobiology. 2014;219(7):512-521.

67. Shaw JM, Trounson AO. Ovarian tissue transplantation and cryopreservation. Application to maintenance and recovery of transgenic and inbred mouse lines. Methods Mol Biol. 2002;180:229-251.

68. Hirota $Y$, et al. The presence of midkine and its possible implication in human ovarian follicles. Am J Reprod Immunol. 2007;58(4):367-373. 